\title{
EFFECT OF INTERFACE TRAPS RELATED TO MOBILE CHARGES ON SILICON n-CHANNEL METAL/OXIDE/SEMICONDUCTOR FIELD EFFECT TRANSISTORS DETERMINED BY A CHARGE-TEMPERATURE TECHNIQUE
}

\author{
J. G. HWU, C. M. LIN AND W. S. WANG
}

Depariment of Electrical Engineering, National Taiwan University, Taipei (Taiwan)

(Received August 13, 1985; revised November 20, 1985; accepted February 11, 1986)

A new charge-temperature technique is proposed which permits control of the motion of mobile charges inside the gate oxide of metal/oxide/semiconductor field effect transistors (MOSFETs). It was shown that the transconductance of an n-channel MOSFET was increased (decreased) after the device was subjected to a negative (positive) charge-temperature treatment. A constant interface trap density model including the cffect of mobility degradation due to interface traps and velocity saturation is developed to explain the experimental results. The interface trap density is increased with the number of mobile charges driven to the $\mathrm{Si}-\mathrm{SiO}_{2}$ interface, whereas the concomitant degradation can be reduced by a suitable negative charge-temperature treatment.

\section{INTRODUCTION}

Device degradation induced by interface traps is very important for the longterm reliability of integrated circuits ${ }^{1,2}$. Since interface traps are closely connected to the insulating layer in metal/oxide/semiconductor field effect transistors (MOSFETs) they are directly controlled by the property of the insulator. There are many reports regarding the formation of interface traps. It can be caused by the injection of hot electrons ${ }^{3}$, by an electric field ${ }^{4}$, by a tunnelling process ${ }^{5}$, by unsatisfied bonds ${ }^{6}$ etc. Wang ${ }^{7}$ and Schulz and Klausmann ${ }^{8}$ have reported the occurrence of interface traps when $\mathrm{Na}^{+}$ions are near the $\mathrm{Si}-\mathrm{SiO}$ interface. Furthermore, Brews and Lope $^{9}$ have reported that the interface trap density $D_{\mathrm{it}}$ is indeed increased when the capacitor is bias-temperature stressed. The actual mechanism of these $\mathrm{Na}^{+}$-related interface traps, however, is still under debate ${ }^{10-12}$.

In this work, an $\mathrm{SiO}_{2}$ layer prepared without any treatment to reduce the mobile charges was used as the gate oxide of an n-channel-(n-) MOSFET. We found that the capacitance-voltage $(C-V)$ curve of the gate capacitor after positive charge-temperature aging was only slightly distorted, as shown in Fig. 1. According to the above reports two mechanisms may be responsible for a $C-V$ distortion. One is the non-uniform distribution of the interface charges ${ }^{10}$ and the other is the increase in $D_{\mathrm{it}}$ which is caused by high field stressing or by mobile charges. In some 


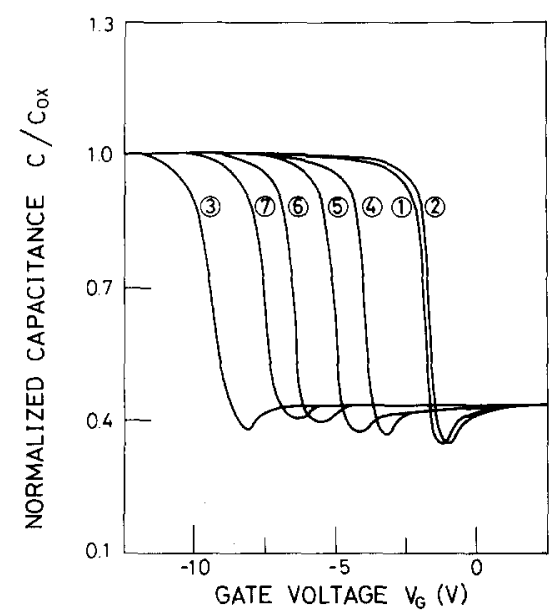

Fig. 1. $C$ - $V$ curves of the gate capacitor of an n-MOSFET before (curve 1) and after charge-temperature treatment at various voltages: curve $2,-9.5 \mathrm{~V}$; curve $3,+9.5 \mathrm{~V}$; curve $4,-4.8 \mathrm{~V}$; curve 5 , $+3.6 \mathrm{~V}$; curve $6,+5.4 \mathrm{~V} ;$ curve $7,+5.8 \mathrm{~V}$

other test runs at our laboratory we found that the $C-V$ curve of an MOS capacitor (not the same as the gate capacitor of the MOSFET) after positive chargetemperature aging was considerably distorted in a certain region which was quite different from that shown in Fig. 1. When frequency dispersion tests ${ }^{13}$ (i.e. the capacitor was tested at 5 and $500 \mathrm{kHz}$ at room temperature) were applied to this sample before and after positive charge-temperature aging, we found that $D_{\text {it }}$ was changed but this did not account for the distortions in the $C-V$ curves mentioned above. We therefore conclude that two mechanisms exist simultaneously. The nonuniformity effect dominates the $C-V$ behaviour in this case and seems to be responsible for the serious distortion of the $C-V$ curve. In the sample investigated in this study, however, the $C-V$ curve is only slightly distorted, as can be seen in Fig. 1; frequency dispersion tests indicated that the remaining distortions are caused only by a change in $D_{\mathrm{it}}$. For example, the dispersion of the capacitances measured at 5 and $500 \mathrm{kHz}$, i.e. $\{C(5 \mathrm{kHz})-C(500 \mathrm{kHz})\} / C_{\mathrm{ox}}$ where $C_{\mathrm{ox}}$ is the oxide capacitance, may exceed about $5 \%$ in the depletion region. Hence, we consider that the fraction of $D_{\text {it }}$ due to the mobile charge is increased when the capacitor is subjected to positive charge-temperature treatment and that it dominates the $C-V$ behaviour of the sample under test. It should be noted that we used the charge-temperature method instead of the conventional bias-temperature method to drive the mobile charges across the oxide. Briefly, the gate capacitor was first charged and then disconnected from the charging system. Since the RC time constant of the gate capacitor is sufficiently high, e.g. greater than $1000 \mathrm{~s}$ at room temperature in our sample, the charges on the metal gate do not disappear quickly. If the capacitor is annealed at $400^{\circ} \mathrm{C}$ in an $\mathrm{N}_{2}$ ambient for about $5 \mathrm{~min}$, the electric field due to these charges will act in the same way as an electric field applied during bias-temperature aging.

We applied the charge-temperature technique to a silicon n-MOSFET and investigated its effect on such characteristics as the current-voltage $(I-V)$ behaviour and the transconductance. Since the quantity of interface traps can be reversibly 
controlled by a suitable charge-temperature treatment, a behaviour once observed can be repeatedly reproduced in the same device. The experimental results indicate that the transconductance is decreased when the number of the interface traps, after positive charge-temperature aging, is increased. A constant $D_{\mathrm{it}}$ model is proposed to explain the experimental results. In this model we consider the effect of velocity saturation, the degradation of mobility due to interface traps ${ }^{14}$, the contact series resistance ${ }^{15}$, and the gate voltage shifts due to the generation of interface-trapped charges and the redistribution of mobile charges. After fitting the experimental $I-V$ data, we find that the fitted $D_{i t}$ is increased according to the number of the mobile charges driven to the $\mathrm{Si}-\mathrm{SiO}_{2}$ interface. These data agree quite well with the results obtained by the $C-V$ measurements. Thus it is believed that the constant $D_{\mathrm{it}}$ model is meaningful in analysing the degradation of MOSFETs as a result of interface traps.

\section{EXPERIMENT}

Since the value of $D_{\mathrm{it}}$ for a $\langle 111\rangle$-oriented wafer is generally greater than that of $a\langle 100\rangle$-oriented wafer, we used a $\langle 111\rangle$-oriented $p$-type silicon wafer with a doping concentration of $3.5 \times 10^{15} \mathrm{~cm}^{-3}$ as the substrate of the n-MOSFET to investigate the effect of interface traps on device degradation. The geometric structure of the device is designed with a channel length $L$ of $15 \mu \mathrm{m}$ and a channel width $Z$ of $400 \mu \mathrm{m}$ to ensure that the one-dimensional approximation was valid. The thickness $d$ of the gate oxide, which was grown by dry oxidization at $1000^{\circ} \mathrm{C}$, is $820 \AA$ in this work. Aluminium was used as the material of the contact electrodes.

The high frequency $(1 \mathrm{MHz}) \mathrm{C}-V$ curves of the gate capacitor after chargetemperature aging at various charging voltages are plotted in Fig. 1. From the figure, it can be seen that the slope of the left-hand $C-V$ curve (curve 3 ) is less than that of the right-hand curve (curve 2). This is due to the increase in the $\mathrm{Si}-\mathrm{SiO}_{2}$ interface traps with the number of mobile charges driven to the interface region.

Immediately after each charge-temperature treatment, the $I-V$ characteristics of the MOSFET are recorded. To minimize the error due to different tests, the testing conditions are set to have the same power limitation of the curve tracer, the same sweep voltage at no device situation and the same range of gate bias voltage $V_{\mathrm{G}}$, i.e. from 0 to $20 \mathrm{~V}$ ( $2 \mathrm{~V}$ per step). Figures 2(a), 2(b) and 2(c) are the $I-V$ curves of the n-MOSFET when it is successively charge-temperature treated at charging voltages $-9.5 \mathrm{~V},+9.5 \mathrm{~V}$ and $-4.8 \mathrm{~V}$ respectively. The corresponding $C-V$ curves of the gate MOS capacitor are shown in Fig. 1 (curves 2, 3 and 4). Since curve 2 shifts to curve 3 after positive charge-temperature aging as shown in Fig. 1, the zero gate bias current shown in Fig. 2(b) (e.g. $I_{\mathrm{D}}=10 \mathrm{~mA}$ at $V_{\mathrm{D}}=6 \mathrm{~V}$, where $I_{\mathrm{D}}$ and $V_{\mathrm{D}}$ are the drain current and voltage with respect to the source) is higher than that shown in Fig. 2(a) (e.g. $I_{\mathrm{D}}=200 \mu \mathrm{A}$ at $\left.V_{\mathrm{D}}=6 \mathrm{~V}\right)$ because of this flat-band voltage shift. In contrast, as $V_{\mathrm{G}}$ increases (e.g. $\left.V_{\mathrm{G}}=20 \mathrm{~V}\right)$, the current shown in Fig. 2(b) (e.g. $I_{\mathrm{D}}=34 \mathrm{~mA}$ at $\left.V_{\mathrm{D}}=6 \mathrm{~V}\right)$ is less than that shown in Fig. $2(\mathrm{a})\left(e . g . I_{\mathrm{D}}=47 \mathrm{~mA}\right.$ at $V_{\mathrm{D}}=6 \mathrm{~V}$ ). The $I-V$ curves shown in Fig. 2(b) appear to be more crowded than those shown in Fig. 2(a), and therefore the transconductance of the n-MOSFET after positive charge-temperature aging is decreased.

If the device is again subjected to negative charge-temperature treatment (e.g. 


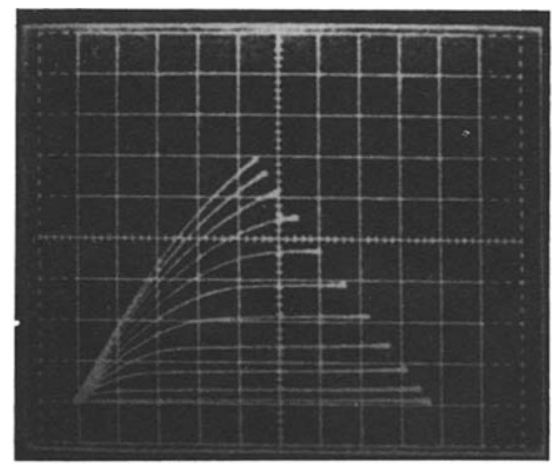

(a)

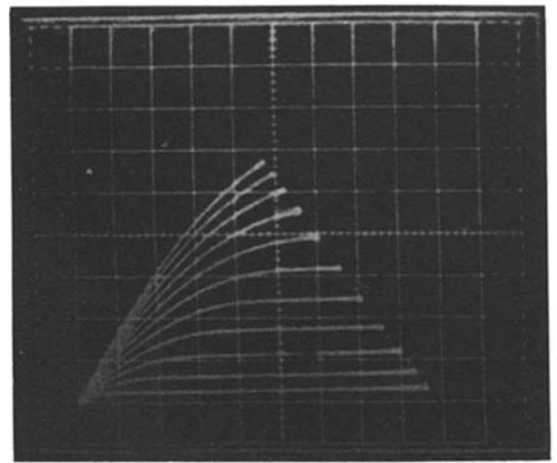

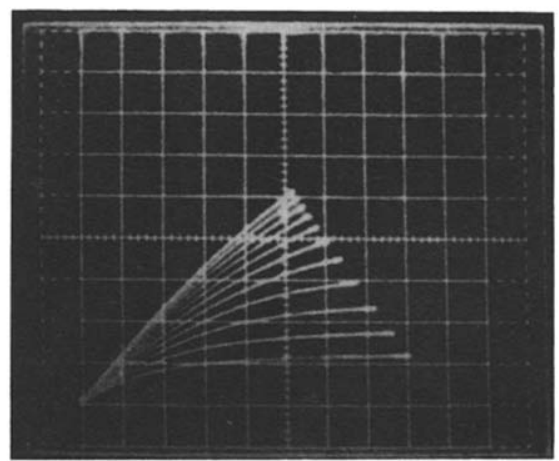

(b)

(c)

Fig. 2. Experimental $\boldsymbol{I}-\boldsymbol{V}$ characteristics of an n-MOSFET after successive charge-temperature agings at charging voltages of $(\mathrm{a})-9.5 \mathrm{~V},(\mathrm{~b})+9.5 \mathrm{~V}$ and $(\mathrm{c})-4.8 \mathrm{~V}$.

$-4.8 \mathrm{~V}$ in this work), the $C-V$ curve shifts from curve 3 to curve 4 in Fig. 1 . Both the $I-V$ characteristic and the transconductance of the device are recovered owing to the decrease in the interface traps, as can be seen from Fig. 2(c). Figure 3 shows the transconductances of the n-MOSFET after various charge-temperature agings corresponding to the $C-V$ curves shown in Fig. 1. It is seen that transconductance degradation is indeed observed when the device is subjected to positive chargetemperature treatment.

It has been reported by Gosney ${ }^{16}$ that there are two contributions to the drainsource leakage current in a MOSFET for a gate voltage below the threshold voltage. One is the reverse bias drain junction leakage current and the other is the surface channel current that flows when the surface is weakly inverted. As the gate voltage is decreased, the drain-source leakage current continues to decrease until no further change occurs in the $I-V$ characteristic and the minimum leakage current $I_{\mathrm{D} 1}$ due to the junction leakage current is achieved. Each time, after the charge-temperature aging, a different $I_{\mathrm{D} 1}$ value is obtained with a different cut-off voltage $V_{\mathrm{G} \text { l }}$ owing to the flat-band shifts. These $I_{\mathrm{D} 1}-V_{\mathrm{D}}$ curves are shown in Fig. 4 . As can be seen from this figure, $I_{\mathrm{D} 1}$ increases as $D_{\mathrm{it}}$ is increased by positive charge-temperature aging. 

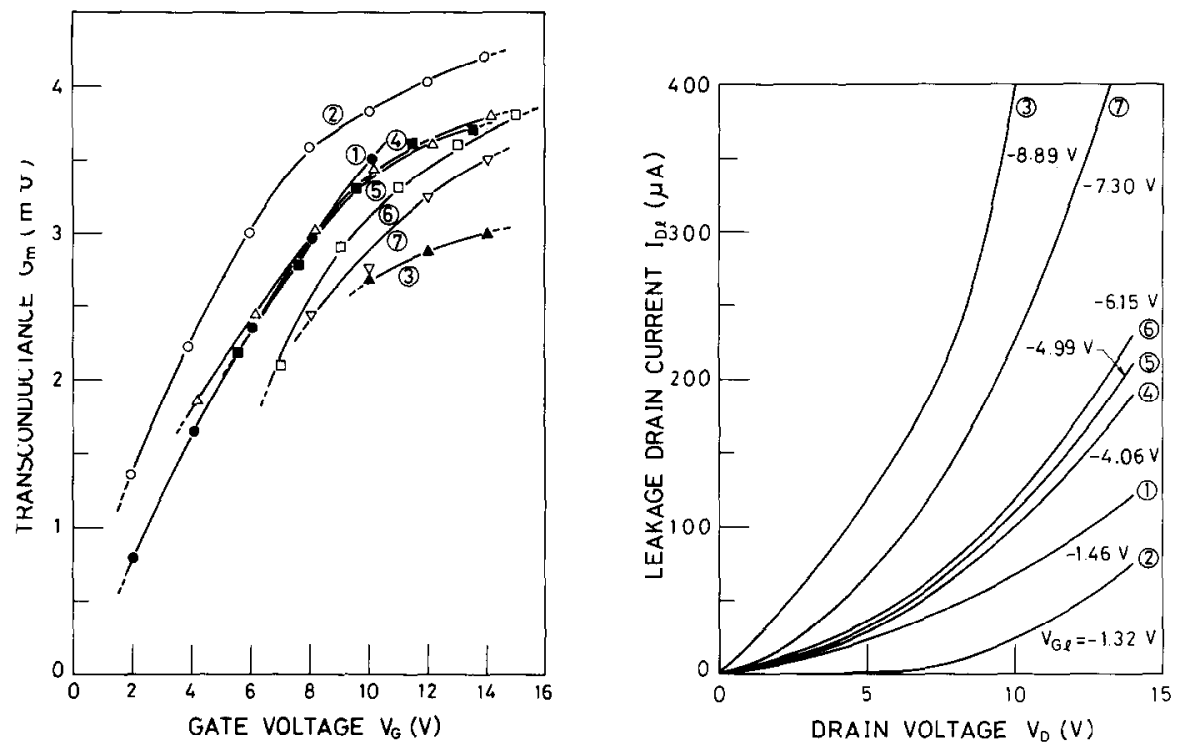

Fig. 3. Transconductances of an n-MOSFET before (curve 1) and after charge-temperature aging at various voltages corresponding to the $C-V$ curves shown in Fig. 1: curve 2, $-9.5 \mathrm{~V}$; curve 3, $+9.5 \mathrm{~V}$; curve $4,-4.8 \mathrm{~V}$; curve $5,+3.6 \mathrm{~V}$; curve $6,+5.4 \mathrm{~V}$; curve $7,+5.8 \mathrm{~V}$.

Fig. 4. Experimental minimum drain leakage current $I_{\mathrm{D} 1} v s$. drain voltage $V_{\mathrm{D}}$, with the cut-off voltage $V_{\mathrm{Gl}}$ as a parameter, before (curve 1 ) and after charge-temperature treatment at various voltages $(d=820 \AA$ ): curve 2, $-9.5 \mathrm{~V}$; curve $3,+9.5 \mathrm{~V}$; curve $4,-4.8 \mathrm{~V}$; curve 5 , $+3.6 \mathrm{~V}$; curve $6,+5.4 \mathrm{~V}$; curve $7,+5.8 \mathrm{~V}$.

\section{MODELLING AND ANALYSIS}

\subsection{Constant $D_{\mathrm{it}}$ approximation}

For an n-MOSFET with $V_{\mathrm{G}}$ and $V_{\mathrm{D}}$, the quasi-Fermi level $E_{\mathrm{Fn}}$ of the minority electron carriers at the point at a distance $y$ from the source electrode along the channel is lowered from the equilibrium Fermi level $E_{\mathrm{Fp}}$ by $E_{\mathrm{Fn}}-E_{\mathrm{Fp}}=-q V(y)$ as shown in Fig. 5, where $V(y)=y V_{\mathrm{D}} / L$ is the reverse bias between point $y$ and the source electrode. First, we assume that $D_{\mathrm{it}}$ is uniformly distributed over the energy band gap. Then the interface trapped charge $Q_{\mathrm{it}}$ of the n-MOSFET at point $y$ can be obtained by integrating $D_{\mathrm{it}}$ from the valence band at the surface to $E_{\mathrm{Fn}}$ as shown in Fig. 5, and is expressed as

$$
Q_{\mathrm{it}}(y)=q D_{\mathrm{it}}\left\{\psi_{\mathrm{s}}(y)-V(y)+0.56-\psi_{\mathrm{B}}\right\}
$$

where $\psi_{\mathrm{S}}(y)$ is the surface potential at point $y$ and $\psi_{\mathrm{B}}$ is the potential difference between the Fermi level and the intrinsic Fermi level at the bulk semiconductor. The charge $Q_{\mathrm{B}}(y)$ within the surface depletion region can be obtained from $\psi_{\mathrm{S}}(y){ }^{17}$ while the charge $Q_{n}(y)$ due to minority carriers within the inversion layer can also be obtained according to charge sheet model ${ }^{18}$. Since $V_{\mathrm{G}}$ is constant for all $y$ values (from the concept of charge neutrality), the values of $V_{\mathrm{G}}, V_{\mathrm{FB}}, Q_{\mathrm{B}}(y), Q_{\mathrm{n}}(y), Q_{\mathrm{it}}(y)$ and $\psi_{\mathrm{s}}(y)$ are correlated, where $V_{\mathrm{FB}}$ is the flat-band voltage.

Several effects on the mobility $\mu_{\mathrm{n}}$ of electrons in n-MOSFETs have been 


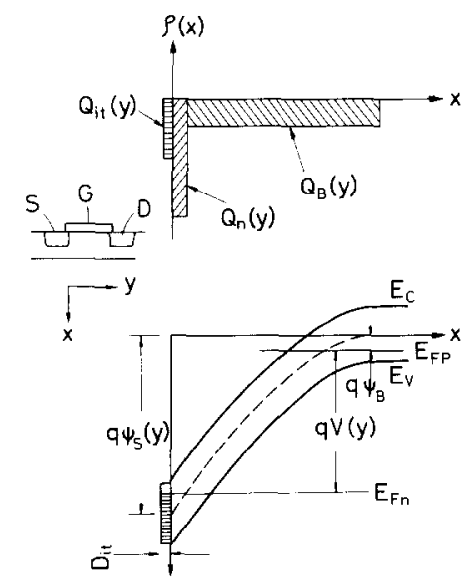

Fig. 5. Comparison of charge distribution and energy band variation in an inverted p region for the nonequilibrium case at a point at a distance $y$ from the source electrode. The constant interface trap density $D_{\mathrm{it}}$ distribution is also shown in this figure.

discussed before. Generally $\mu_{\mathrm{n}}$ is a function of the low field mobility $\mu_{\mathrm{no}}$ and the saturation velocity ${ }^{19}$. In addition, the mobility degradation as a function of $D_{\mathrm{it}}$ is also important and can be expressed by the empirical relationship ${ }^{1,14}$

$$
\mu_{\mathrm{n} 0}=\frac{\mu_{0}}{1+\alpha N_{\mathrm{it}}}
$$

where $\mu_{0}=3490-164 \lg N_{\mathrm{A}}=940, \alpha=-0.104+0.0193 \lg N_{\mathrm{A}}=0.196$ and $N_{\mathrm{it}}$ is the total number of interface traps in units of $10^{11} \mathrm{~cm}^{-2}$. Since we have assumed that $D_{\mathrm{it}}$ is constant for the energy levels within the silicon band gap (i.e. $\left.1.12 \mathrm{eV}\right), N_{\mathrm{it}}$ can be obtained as $N_{\mathrm{it}}=1.12 D_{\mathrm{it}}$. From the above considerations, we can then evaluate $I_{\mathrm{D}}$ numerically.

Apart from considering the effect of $D_{\mathrm{it}}$ on $I_{\mathrm{D}}$, the contact series resistance $R_{0}$ is also considered in this analysis. The relationship between $I_{\mathrm{D}}$ and $V_{\mathrm{D}}$ should then be modified as $V_{\mathrm{D}}{ }^{\prime}=V_{\mathrm{D}}+I_{\mathrm{D}} R_{0}$, where $V_{\mathrm{D}}{ }^{\prime}$ is the applied drain voltage.

\subsection{Analysis}

If the experimental $I-V$ curves are optimally fitted by the theory mentioned above, two fitted parameters, i.e. $D_{\mathrm{it}}$ and $R_{0}$, can be obtained. Seven sets of the fitted curves and the experimental $I-V$ curves at $V_{\mathrm{G}}=8 \mathrm{~V}$ are plotted in Fig. 6 for comparison. Annealing at $400^{\circ} \mathrm{C}$ make the contacts better, so $R_{0}$ is smaller after than before annealing. Typical values of $R_{0}$ are $210 \Omega$ before annealing and $70 \Omega$ after annealing. As can be seen from this figure, the fitted results are quite consistent with the experimental curves.

Since the redistribution of the mobile charges inside the oxide after various charge-temperature agings may result in the shifts in the $C-V$ curves shown in Fig. 1, the change $\Delta N_{\mathrm{m}, \text { eff }}$ in the effective mobile charge with respect to curve 2 in Fig. 1 can be obtained from

$$
\Delta N_{\mathrm{m}, \mathrm{eff}}=C_{\mathrm{ox}} \Delta V_{\mathrm{FB}} / q
$$


where $\Delta V_{\mathrm{FB}}$ is the shift in the flat-band voltage with respect to the reference $C-V$ curve (curve 2 in Fig. 1). Meanwhile, if the fitted $D_{i t}$ value of curve 2 in Fig. 6 is taken as a reference, the change $\Delta D_{\mathrm{it}}$ in the interface trap density can also be obtained and plotted versus $\Delta N_{\mathrm{m}, \text { eff }}$, as shown in Fig. 7. Also shown in this figure are the $C-V$ measured data (average value) obtained by the Terman method ${ }^{20}$. As can be seen from this figure, $\Delta D_{\mathrm{it}}$ increases with $\Delta N_{\mathrm{m} \text {,eff }}$ according to both the fitted $I-V$ data and the measured $C-V$ data.
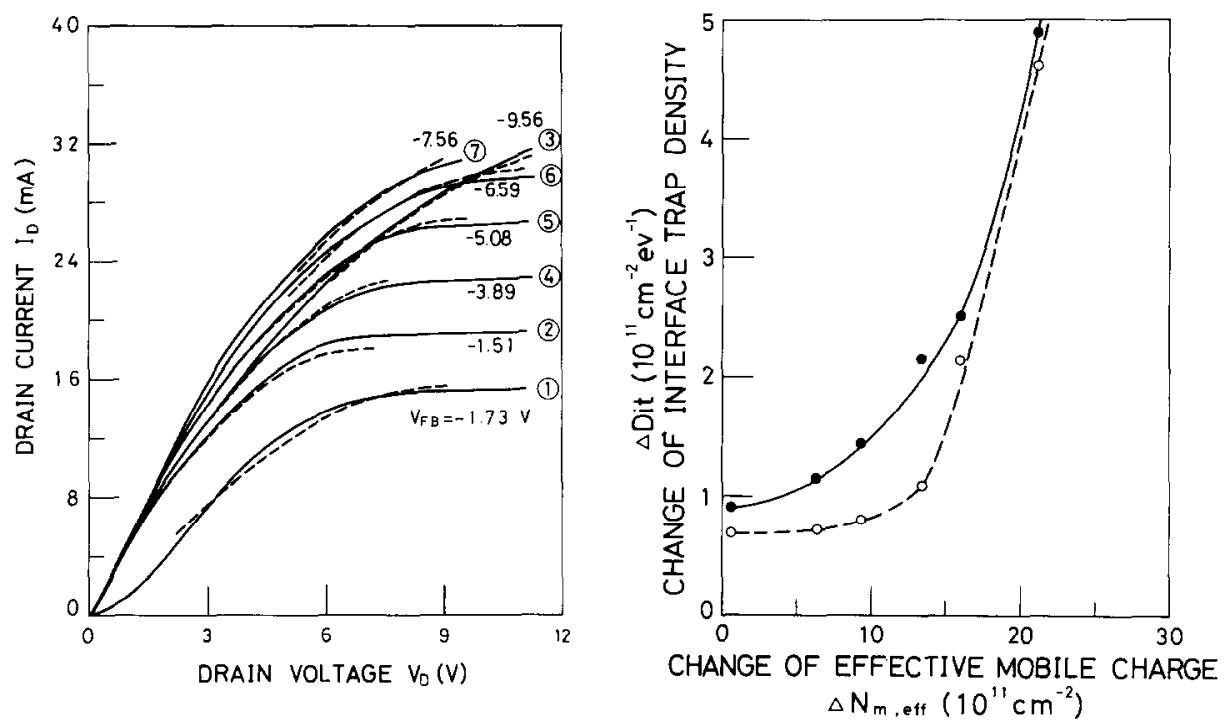

Fig. 6. Theoretical curve fittings of the experimental $I-V$ characteristics $\left(V_{G}=8 \mathrm{~V}\right)$ for an n-MOSFET after charge-temperature aging at various voltages corresponding to the $C-V$ curves shown in Fig. 1. Measured curves (-) $\left(V_{\mathrm{G}}=8 \mathrm{~V}\right)$ before (curve 1) and after annealing at various voltages: curve 2 , $-9.5 \mathrm{~V}$; curve 3 , +9.5 V; curve $4,-4.8 \mathrm{~V}$; curve 5 , $+3.6 \mathrm{~V}$; curve 6 , + $5.4 \mathrm{~V}$; curve 7 , + $5.8 \mathrm{~V}$. Fitted curves $(--)$ for various values of $D_{\mathrm{it}}\left(\times 10^{11} \mathrm{~cm}^{-2} \mathrm{eV}^{-1}\right)$ : curve $1,1.2$; curve $2,0.3$; curve $3,5.2$; curve 4 , 1.45 ; curve $5,1.75$; curve $6,2.45$; curve $7,2.8$. The values of $R_{0}$ were $210 \Omega$ for curve 1 and $70 \Omega$ for curves $2-7$.

Fig. 7. Relationship between the change $\Delta D_{\mathrm{it}}$ in interface trap density $v$ s. the change $\Delta N_{\mathrm{m}, \text { eff }}$ in effective mobile charge for both the fitted $I-V$ data $(\bullet)$ and the measured $C-V$ data $(O)$ (determined by the Terman method).

\section{DISCUSSION}

From Figs. 1 and 3 the transconductance (curve 2 in Fig. 3) of the n-MOSFET reaches its maximum value when the device is annealed with a negative chargetemperature aging process. In this case, most of the mobile charges are drawn to the metal-oxide interface, so their effects on the formation of the interface traps near the $\mathrm{Si}-\mathrm{SiO}_{2}$ interface are minimal; therefore the channel mobility is maximal.

When the mobile charges are driven to the $\mathrm{Si}-\mathrm{SiO}_{2}$ interface by positive charge-temperature aging, the corresponding $C-V$ curve shifts to the left $(e . g$. curves 3-7 in Fig. 1). Now the effect of mobile charges on the formation of interface traps is increased, and therefore the channel mobility is degraded. As can be seen 
from curves 3-7 in Fig. 3, the transconductances are gradually decreased owing to this effect.

In Fig. 7, the $I-V$-fitted $\Delta D_{\mathrm{it}}$ is much closer to the $C-V$-measured $\Delta D_{\mathrm{it}}$ at large $\Delta N_{\mathrm{m}, \text { eff }}$ than at small $\Delta N_{\mathrm{m} \text {,eff }}$. Because the $C-V$-measured $\Delta D_{\mathrm{it}}$ is the average value of $\Delta N_{\text {it }}$ within the energy levels which are obtainable by the Terman method while the $I-V$-fitted $\Delta D_{\mathrm{it}}$ is the actual value for the device when it is operated under strong inversion condition, so the first value can be regarded as that of $\Delta D_{\mathrm{it}}$ near the midgap while the second value gives $\Delta D_{\mathrm{it}}$ near the conduction band. Since the quantity of interface traps can be reduced after negative charge-temperature aging (i.e. for the low $\Delta N_{\mathrm{m}, \text { cff }}$ situation), the $D_{\mathrm{it}}$ distribution of the $\mathrm{n}$-MOSFET is somewhat like a U-shaped curve ${ }^{21}$. The value of $D_{\mathrm{it}}$ near the midgap is smaller than that near the conduction band. Hence $\Delta D_{i t}$ values mismatch in this case. In contrast, if the device is subjected to positive charge-temperature treatment (i.e. for the high $\Delta N_{\text {m.eff }}$ situation), the $D_{\mathrm{it}}$ value near the midgap increases and becomes close to that near the conduction band. Therefore the corresponding $\Delta D_{\mathrm{it}}$ values are close to each other. The same behaviour of $\Delta D_{\mathrm{it}}$ is also observed in our samples with different oxide thicknesses. Nevertheless, the trend of the increase in $\Delta D_{\mathrm{it}}$ with increasing $\Delta N_{\mathrm{m}, \mathrm{eff}}$ is observed from the curves in Fig. 7, which is consistent with the actual behaviour of the transconductance (i.e. Fig. 3). Hence we suggest that the constant $D_{\mathrm{it}}$ model is good enough in the analysis of $D_{\mathrm{it}}$ evaluation.

\section{CONCLUSION}

Charge-temperature aging provides a very convenient and efficient way of controlling the quantity of interface traps in not only an MOS capacitor but also an MOSFET. In particular, the transconductance of an MOSFET is found to be increased (decreased) after the device is annealed with negative (positive) chargetemperature aging. The constant $D_{\mathrm{it}}$ model gives a good explanation of the degradation of n-MOSFET characteristics, and is expected to be useful in some design considerations of MOSFET.

\section{REFERENCES}

1 F. C. Hsu and S. Tam, IEEE Electron Device Lett., 5 (1984) 50.

2 D. L. Lile, Solid-State Electron., 21 (1978) 1199.

3 B. Borchert, K. R. Hofmann and G. Dorda, Electron. Lett., 19 (1983) 746.

4 J. L. Crowley, H. J. Hoffman and T. J. Stultz, J. Appl. Phys., 53 (1982) 6919.

5 B. Eitan, D. Frohamn-Bentchkowsky, J. Shappir and M. Balog, Appl. Phys. Lett., 40 (1982) 523.

6 B. E. Deal, J. Electrochem. Soc., 121 (1974) 198C.

7 K. L. Wang, in H. R. Huff and E. Sirtl (eds.), Semiconductor Silicon, Electrochemical Society, Princeton, NJ, 1977, p. 404.

8 M. Schulz and E. Klausmann, Appl. Phys., 18 (1979) 169.

9 J. R. Brews and A. D. Lopez, Solid-State Electron., 16 (1973) 1267.

10 D. J. Silversmith, J. Electrochem. Sac., 110(1972) 1589.

11 D. J. DiMaria, J. M. Aitken and D. R. Young, J. Appl. Phys., 47 (1976) 2740.

12 W. W. Hillen and D. G. Hemmes, Solid-State Electron., 24 (1981) 773.

13 C. C. Chang and W. C. Johnson, IEEE Trans. Electron Devices, 24 (1977) 1249.

14 S. C. Sun and J. D. Plummer, IEEE Trans. Electron Devices, 27 (1980) 1497. 
15 M. H. Seavey, IEEE Electron Device Lett., 5 (1984) 479.

16 W. M. Gosney, IEEE Trans. Electron Devices, 19 (1972) 213.

17 S. M. Sze, Physics of Semiconductor Devices, Wiley, New York, 2nd edn., 1981, p. 439.

18 J. R. Brews, Solid-State Electron., 21 (1978) 345.

19 C. G. Sodini, P.-K. Ko and J. L. Moll, IEEE Trans. Electron Devices, 31 (1984) 1386.

20 L. M. Terman, Solid-State Electron., 5 (1962) 285.

21 T. Sakurai and T. Sugano, J. Appl. Phys., 52 (1981) 2889. 